\title{
Investigation of Order among Some Known T-norms
}

\author{
Shohel Babu' ${ }^{1}$, Fatema Tuj Johora ${ }^{1}$, Abdul Alim² \\ ${ }^{1}$ Mathematics, IUBAT-International University of Business Agriculture and Technology, Dhaka, Bangladesh \\ ${ }^{2}$ Mathematics, BGMEA University of Fashion and Technology, Dhaka, Bangladesh
}

\section{Email address:}

shohel.babu@iubat.edu (S. Babu), fatema.johorea@iubat.edu (F. T. Johora), abdulalim@buft.edu.bd (A. Alim)

\section{To cite this article:}

Shohel Babu, Fatema Tuj Johora, Abdul Alim. Investigation of Order among Some Known T-norms. American Journal of Applied Mathematics. Vol. 3, No. 5, 2015, pp. 229-232. doi: 10.11648/j.ajam.20150305.14

\begin{abstract}
In this paper, order among some known T-norms is investigated. Firstly, the T-norm which is the strongest or greatest and the T-norm which is the weakest is observed. Comparing two T-norms we establish the relation which is strong or weak. In addition, for parametric T-norms after changing the interval of their parameter a relation has established which is strong or weak. Finally, compared has done among three or more T-norms.
\end{abstract}

Keywords: $\mathrm{T}$-norm Algebraic Product $\mathrm{T}_{\mathrm{P}}$, T-norm $\mathrm{Min}_{\mathrm{M}}$, $\mathrm{T}$-norm Drastic Product $\mathrm{T}_{\mathrm{D}}$, T-norm Franks Product $\mathrm{T}_{\mathrm{F}}$, T-norm Einstein Product $\mathrm{T}_{\mathrm{E}}$, T-norm Hamacher Product $\mathrm{T}_{\mathrm{H}}$, T-norm Dubois \& Prade Product $\mathrm{T}_{\mathrm{DP}}$

\section{Introduction}

T-norms or triangular norms are generalization of the classical triangular inequality according to $\mathrm{K}$. Menger in 1942. In 1960, B. Schweizer and A. Sklar after revision of this work redefined the concept of triangular norm as an associative and commutative binary operation. They play a fundamental role in probabilistic metric spaces, probabilistic norms and scalar products, multiple-valued logic, fuzzy sets theory.

\section{Definition:}

A T-norm is a functionT: $[0,1] \times[0,1] \rightarrow[0,1]$ which satisfies the following properties:

$\left[T_{1}\right]$ :Monotonicity: $T(a, b) \leq T(c, d)$ if $a \leq$ cand $b \leq d$

$\left[T_{2}\right]$ :Commutativity: $T(a, b)=T(b, a)$

$\left[T_{3}\right]$ :Associativity: $T(a, T(b, c))=T(T(a, b), c)$

$\left[T_{4}\right]$ :Boundary condition: $T(a, 1)=a$

\section{Ordering of T-norms}

\subsection{Proposition: Among all T-norm "Min" is the Strongest}

Proof: Suppose T is any T-norm.

$\forall x, y \in \mathrm{I}$;

$T(x, y) \leq T(x, 1)$ [by boundary condition]

$=x$ [by boundary condition]

So $T(x, y) \leq x$.

Again $T(x, y) \leq T(y, x)$ [by commutativity]

$\leq T(y, 1)$ [by monotonicity] $=y$.

So $T(x, y) \leq y$.

Consequently, $T(x, y)$ is a lower bound of $\{x, y\}$.

Again min $(x, y)$ is the greatest lower bound (glb) of $\{x, y\}$.

But $\mathrm{I}$ is in chain, so $\mathrm{T}_{\mathrm{M}}(x, y) \geq T(x, y)$.

Hence "min" is the strongest or greatest t-norm.

\subsection{Proposition: Among all T-norm "Drastic Product" is the Weakest}

Proof: Suppose T is any T-norm.

$\forall x, y \in \mathrm{I}$; there are two cases:

Case1: Without loss of generality suppose $x=1$, then $T_{D}(x, y)=T_{D}(1, y)=y$ and $T(x, y)=T(1, y)=y$ [by boundary condition]. Consequently $T_{D}=1$.

Case2: When $x \neq 1$ and $y \neq 1$, then

$\mathrm{T}_{\mathrm{D}}(\mathrm{x}, \mathrm{y})=0$.

But in I; clearly, $0 \leq T(x, y)$

Therefore $T_{D}(x, y) \leq T(x, y)$.

Hence among all T-norm $T_{D}$ is the weakest.

\subsection{Proposition: Let ke]0, 1 [ , Frank's Product}

$$
T_{F}(x, y)_{k}=\log _{k}\left(1+\frac{\left(k^{x}-1\right)\left(k^{y}-1\right)}{k-1}\right)
$$

And Algebraic Product $T_{P}(x, y)=x y, \forall(x, y) \in I \times I$. Then $T_{F}(x, y)_{k} \geq T_{P}(x, y), \forall(x, y) \in I \times I$.

Proof: Let $x \in] 0,1\left[\right.$ and $R_{x}:[0,1] \rightarrow \mathbb{R}: R_{x}(v)=$ $x v^{x-1}, \forall v \in[0,1]$. 
Then $R_{x}(v) \geq 0$, and $\frac{\delta}{\delta x}\left(R_{x}\right)=x(x-1) v^{x-2} \leq 0$, which means that $R_{x}(v)$ is monotonically decreasing.

If we have $U_{x}(v)=v^{x}$, then by mean value theorem we get

$\frac{U_{x}(1)-U_{x}(v)}{1-v}=\frac{\delta}{\delta x} U_{x}\left(\xi_{0}\right)$

$=R_{x}\left(\xi_{0}\right)$, because $\frac{\delta}{\delta x} U_{x}(v)=x v^{x-1}$

$\left.\leq \mathrm{R}_{\mathrm{x}}(\mathrm{v}), \xi_{0} \epsilon\right] \mathrm{v}, 1[$.

i.e.

$\left.\frac{1-\mathrm{v}^{\mathrm{x}}}{1-\mathrm{v}} \leq \mathrm{xv}^{\mathrm{x}-1}, \forall \mathrm{x}, \mathrm{v} \epsilon\right] 0,1[$.

Now let $H_{x}:[0,1] \rightarrow \mathbb{R}$,

$\left.\mathrm{H}_{\mathrm{x}}(\mathrm{v})=\frac{1-\mathrm{v}^{\mathrm{x}}}{1-\mathrm{v}}, \mathrm{v} \epsilon\right] 0,1[$.

Here

$\left.\frac{\delta}{\delta \mathrm{x}} \mathrm{H}_{\mathrm{x}}(\mathrm{v})=\frac{-\mathrm{xv}^{\mathrm{x}-1}(1-\mathrm{v})+1-\mathrm{v}^{\mathrm{x}}}{(1-\mathrm{v})^{2}} \leq 0, \forall \mathrm{x}, \mathrm{v} \epsilon\right] 0,1[$.

Which means that $H_{x}(v)$ is monotonically decreasing and, Furthermore,

$\left.\mathrm{H}_{\mathrm{x}}(\mathrm{k}) \leq \mathrm{H}_{\mathrm{x}}\left(\mathrm{k}^{\mathrm{y}}\right), \forall \mathrm{k}, \mathrm{y} \epsilon\right] 0,1[$,

i.e

$\frac{1-k^{x}}{1-k} \geq \frac{1-k^{x y}}{1-k^{y}}$

$\Rightarrow \frac{k^{x}-1}{k-1} \geq \frac{k^{x y}-1}{k^{y}-1}$

$\Rightarrow \frac{\left(k^{x}-1\right)\left(k^{y}-1\right)}{k-1} \leq k^{s y}-1\left[\because k^{y}-1 \leq 0\right]$

$\Rightarrow 1+\frac{\left(k^{x}-1\right)\left(k^{y}-1\right)}{k-1} \leq k^{s y}$

$\Rightarrow 1+\frac{\left(k^{x}-1\right)\left(k^{y}-1\right)}{k-1} \leq k^{s y}$

$\Rightarrow \log _{k}\left(1+\frac{\left(k^{x}-1\right)\left(k^{y}-1\right)}{k-1}\right) \geq \log _{k}\left(k^{s y}\right)=x y$.

This means that $\left.T_{F}(x, y)_{k} \geq T_{P}(x, y), \forall k, x, y \epsilon\right] 0,1[$.

Again, we clam that

$T_{F}(0, x)_{k}=T_{F}(x, 0)_{k}=T_{P}(0, x)=T_{P}(x, 0)=0$.

And

$T_{F}(x, 1)_{k}=T_{F}(1, x)_{k}=T_{P}(x, 1)=T_{P}(1, x)$

$$
=x, \forall k, x, \epsilon] 0,1[\text {. }
$$

Hence $T_{F}(x, y)_{k} \geq T_{A}(x, y), \forall(x, y) \in I \times I$.

\subsection{Proposition: Let ke]1, $\infty[$, Frank's Product}

$(x, y)_{k}=\log _{k}\left(1+\frac{\left(k^{x}-1\right)\left(k^{y}-1\right)}{k-1}\right)$

and Algebraic Product $T_{P}(x, y)=x y, \forall(x, y) \in I \times I$.

Then $T_{F}(x, y)_{k} \leq T_{P}(x, y), \forall(x, y) \epsilon I \times I$.

Proof: Let $x \in] 0,1\left[\right.$ and $R_{x}:[1, \infty] \rightarrow \mathbb{R}: R_{x}(v)=$ $x v^{x-1}, \forall v \in[0,1]$.

Then $R_{x}(v) \geq 0$, and $\frac{\delta}{\delta x}\left(R_{x}\right)=x(x-1) v^{x-2} \leq 0$, which means that $R_{x}(v)$ is monotonically decreasing.

Now we have,

$U_{x}:\left[1, \infty\left[\rightarrow \mathbb{R}, U_{x}(v)=v^{x}, \forall x \in\right] 0,1[\right.$ and

$\frac{U_{x}(v)-U_{x}(1)}{v-1}=\frac{\delta}{\delta x} U_{x}\left(\xi_{0}\right)$

$\left.=R_{x}\left(\xi_{0}\right), \forall \xi_{0} \epsilon\right] 0,1[\forall$

$\geq R_{x}(v)$

because $R_{x}(v)$ is monotonically decreasing and $\xi_{0} \leq v$. i.e.

$$
\left.\frac{v^{x}-1}{v-1}=x \xi_{0}^{x-1}, \forall x \epsilon\right] 0,1[, v \epsilon] 1, \infty[
$$

Now let $H_{x}(v):[1, \infty] \rightarrow \mathbb{R}$,

$\left.\mathrm{H}_{\mathrm{x}}(\mathrm{v})=\frac{\mathrm{v}^{\mathrm{x}}-1}{\mathrm{v}-1}, \forall \mathrm{v} \epsilon\right] 1, \infty[, \mathrm{x} \epsilon] 0,1[$

We see that

$\frac{\delta}{\delta \mathrm{x}} \mathrm{H}_{\mathrm{x}}(\mathrm{v})=\frac{\mathrm{xv}^{\mathrm{x}-1}(\mathrm{v}-1)-\mathrm{v}^{\mathrm{x}}+1}{(\mathrm{v}-1)^{2}} \leq 0$

According to (1) and $H_{x}(v)$ is monotonically decreasing.

Then,

$\left.\mathrm{H}_{\mathrm{x}}(\mathrm{k}) \leq \mathrm{H}_{\mathrm{x}}\left(\mathrm{k}^{\mathrm{y}}\right), \forall \mathrm{y} \epsilon\right] 0,1[\mathrm{k} \epsilon] 1, \infty[$.

i.e.

$$
\frac{k^{x}-1}{k-1} \geq \frac{k^{x y}-1}{k^{y}-1}
$$

From (2) we get

$$
\begin{aligned}
& 1+\frac{\left(\mathrm{k}^{\mathrm{x}}-1\right)\left(\mathrm{k}^{\mathrm{y}}-1\right)}{\mathrm{k}-1} \leq \mathrm{k}^{\mathrm{sy}} \\
& \Rightarrow \log _{\mathrm{k}}\left(1+\frac{\left(\mathrm{k}^{\mathrm{x}}-1\right)\left(\mathrm{k}^{\mathrm{y}}-1\right)}{\mathrm{k}-1}\right) \leq \log _{\mathrm{k}}\left(\mathrm{k}^{\mathrm{xy}}\right) \\
& \Rightarrow \log _{\mathrm{k}}\left(1+\frac{\left(\mathrm{k}^{\mathrm{x}}-1\right)\left(\mathrm{k}^{\mathrm{y}}-1\right)}{\mathrm{k}-1}\right) \leq \mathrm{xy} .
\end{aligned}
$$

This means that $T_{F}(x, y)_{k} \leq T_{P}(x, y), \forall(x, y) \in I \times I$, $\forall k \epsilon] 1, \infty[$.

$$
\text { Moreover }_{F}(0, x)_{k}=T_{F}(x, 0)_{k}=T_{P}(0, x)
$$

And

$$
=T_{P}(x, 0)=0
$$

$\mathrm{T}_{\mathrm{F}}(\mathrm{x}, 1)_{\mathrm{k}}=\mathrm{T}_{\mathrm{F}}(1, \mathrm{x})_{\mathrm{k}}=\mathrm{T}_{\mathrm{P}}(\mathrm{x}, 1)=\mathrm{T}_{\mathrm{P}}(1, \mathrm{x})$

$$
=\mathrm{x}, \forall \mathrm{x} \epsilon] 0,1[, \forall \mathrm{k}, \epsilon] 1, \infty[\text {. }
$$

Hence $\left.T_{F}(x, y)_{k} \leq T_{P}(x, y), \forall(x, y) \epsilon I \times I, \forall k \epsilon\right] 1, \infty[$.

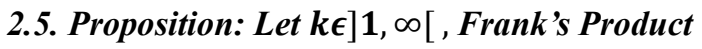

$$
T_{F}(x, y)_{k}=\log _{k}\left(1+\frac{\left(k^{x}-1\right)\left(k^{y}-1\right)}{k-1}\right)
$$

And Boundary Product

$$
T_{B}(x, y)=(0 \wedge x+y-1), \forall(x, y) \epsilon I \times I .
$$

Then $T_{B}(x, y) \leq T_{F}(x, y)_{k}, \forall(x, y) \epsilon I \times I$.

Proof: We will distinguish two cases:

Case1: If $x+y \geq 1$ then $T_{B}(x, y)=x+y-1$

Now for $T_{B}(x, y) \leq T_{F}(x, y)_{k}$ we get

$$
x+y-1 \leq \log _{k}\left(1+\frac{\left(k^{x}-1\right)\left(k^{y}-1\right)}{k-1}\right)
$$

$\Leftrightarrow k^{x+y-1} \leq 1+\frac{\left(k^{x}-1\right)\left(k^{y}-1\right)}{k-1}$

$\Leftrightarrow k^{x+y-1}-1 \leq \frac{\left(k^{x}-1\right)\left(k^{y}-1\right)}{k-1}$

$\Leftrightarrow\left(k^{x+y-1}-1\right)(k-1) \leq\left(k^{x}-1\right)\left(k^{y}-1\right)$

$\Leftrightarrow k^{x+y}-k-k^{x+y-1}+1 \leq k^{x+y}-k^{x}-k^{y}+1$

$\Leftrightarrow k^{x}+k^{y} \leq k+k^{x+y-1}$

$\Leftrightarrow k \cdot k^{x-1}+k \cdot k^{y-1} \leq k+k \cdot k^{(x-1)(y-1)}$

$\Leftrightarrow k^{x-1}+k^{y-1} \leq 1+k^{(x-1)(y-1)}$

$\Leftrightarrow 1-k^{x-1}-k^{y-1}+k^{(x-1)(y-1)} \geq 0$

$\Leftrightarrow 1\left(1-k^{x-1}\right)-k^{y-1}\left(1-k^{x-1}\right) \geq 0$

$\Leftrightarrow\left(1-k^{x-1}\right)\left(1-k^{y-1}\right) \geq 0$

Which is true, because $k>1, x-1 \leq 0, y-1 \leq 0$ and 
$k^{x-1} \leq k^{0}=1$

$k^{y-1} \leq k^{0}=1$.

Case2: If $x+y<1$ then

$T_{B}(x, y)=0$

$\operatorname{For}_{B}(x, y) \leq T_{F}(x, y)_{k} \Rightarrow 0 \leq T_{F}(x, y)_{k}$

This is obvious.

Hence $\left.T_{B}(x, y) \leq T_{F}(x, y)_{k}, \forall(x, y) \epsilon I \times I, k \epsilon\right] 1, \infty[$.

\subsection{Proposition: Let $k \epsilon[0,1]$, Duboi's \& Prade Product}

$$
T_{D P}(x, y)_{k}=\frac{x y}{x \vee y \vee k}
$$

and Algebrai Product $T_{P}(x, y)=x y, \forall(x, y) \epsilon I \times I$.

Then $T_{P}(x, y) \leq T_{D P}(x, y), \forall(x, y) \epsilon I \times I, \forall(x, y) \epsilon I \times I$.

Proof: We will distinguish three cases, according to the maximum value of $x$, yandk.

Caes1: If $x \vee y \vee k=x$, then

$T_{D P}(x, y)_{k}=\frac{x y}{x}$

$=y$

$\geq x y,[\therefore x, y \in I]$

$=T_{P}(x, y)$.

$\therefore T_{P}(x, y) \leq T_{D P}(x, y)_{k}$.

Case 2: If $x \vee y \vee k=y$, then

$T_{D P}(x, y)_{k}=\frac{x y}{y}$

$=x$

$\geq x y,[\therefore x, y \in I]$

$=T_{P}(x, y)$.

$\therefore T_{P}(x, y) \leq T_{D P}(x, y)_{k}$.

Case3: If $x \vee y \vee k=k$, then

$T_{D P}(x, y)_{k}=\frac{x y}{k}$

$=\frac{1}{k}(x y)$

$=\frac{1}{k} T_{P}(x, y)$

$\geq T_{a}(x, y)$.

$\therefore T_{P}(x, y) \leq T_{D P}(x, y)_{k}, \forall(x, y) \epsilon I \times I, \forall k \epsilon[0,1]$.

Hence we can say from three cases $T_{P}(x, y) \leq T_{D P}(x, y)_{k}$.

\subsection{Proposition: Let Einstein Product $T_{E}: I \times I \rightarrow I$}

$$
T_{E}(x, y)=\frac{x y}{1+(1-x)(1-y)}
$$

Boundery Produuct $T_{B}(x, y)=0 \mathrm{~V} x+y-1$,

Algebraicc Prodducct $T_{P}(x, y)=x y, \forall(x, y) \in I \times I$.

Then $T_{B}(x, y) \leq T_{E}(x, y) \leq T_{P}(x, y), \forall(x, y) \epsilon I \times I$.

Proof: Now we will split the proof into two cases:

Case1: If $T_{E}(x, y) \leq T_{P}(x, y)$, then

$$
\begin{aligned}
& \frac{x y}{1+(1-x)(1-y)} \leq x y \\
& \Leftrightarrow \frac{x y}{1+(1-x)(1-y)} \leq 1 \\
& \Leftrightarrow 1+(1-x)(1-y) \geq 1 \\
& \Leftrightarrow(1-x)(1-y) \geq 0
\end{aligned}
$$

Which is true, because $\forall(x, y) \epsilon I \times I$.

Case 2: If $T_{B}(x, y) \leq T_{E}(x, y)$, then

$$
0 \mathrm{~V}(x+y-1) \leq \frac{x y}{1+(1-x)(1-y)} \text {. }
$$

(i)If $x+y \leq 1$, then $0 \mathrm{~V}(x+y-1)=0$.

In this case we get,

$$
0 \leq \frac{x y}{1+(1-x)(1-y)}
$$

Since $x, y \in[0,1]$, soxy $\geq 0$ and $\frac{x y}{1+(1-x)(1-y)} \geq 0$

$\therefore T_{B}(x, y) \leq T_{E}(x, y)$

(i)Otherwise $x+y \geq 1$, then $0 \mathrm{~V}(x+y-1)=x+y-$ 1.

$(x+y-1) \leq \frac{x y}{1+(1-x)(1-y)}$

$\Leftrightarrow(x+y-1)(1-x)(1-y) \leq x y$

$\Leftrightarrow x y-(x+y-1)(1-x)(1-y) \geq 0$

$\Leftrightarrow x y-(x+y-1)(2-x-y+x y) \geq 0$

$\Leftrightarrow x y-\left(2 x-x^{2}-x y+x^{2} y+2 y-x y-y^{2}+x y^{2}-2\right.$ $+x+y-x y) \geq 0$

$\Leftrightarrow x y-\left(3 x-x^{2}+x^{2} y-3 x y+3 y-y^{2}+x y^{2}-2\right) \geq 0$

$\left.\Leftrightarrow x y-3 x+x^{2}-x^{2} y+3 x y-3 y+y^{2}-x y^{2}+2\right) \geq 0$

$\Leftrightarrow x^{2}(1-y)-3 x+4 x y-x y^{2}+2-3 y+y^{2} \geq 0$

$\Leftrightarrow x^{2}(1-y)-x\left(3-4 y+y^{2}\right)+(2-y)(1-y) \geq 0$

$\Leftrightarrow x^{2}(1-y)-x(3-y)(1-y)+(1-y 0(2-y) \geq 0$

$\Leftrightarrow(1-y)\left(x^{2}-x(3-y)+2-y\right) \geq 0$

$\Leftrightarrow(1-y)\left(x^{2}-3 x+x y+2-y\right) \geq 0$

$\Leftrightarrow(1-y)\left(2-2 x-x+x^{2}-y+x y\right) \geq 0$

$\Leftrightarrow(1-y)(1-x)(2-x-y) \geq 0$.

This is true $\forall x, y \in[0,1]$.

From above we may conclude that

$$
T_{B}(x, y) \leq T_{E}(x, y) \leq T_{P}(x, y), \forall(x, y) \in I \times I .
$$

\subsection{Proposition:Let Hamacher Product $T_{H}: I \times I \rightarrow I$}

$$
T_{H}(x, y)_{k}=\frac{x y}{k+(1-k)(x+y-x y)}, k \in[1,2] .
$$

Then $T_{B}(x, y) \leq T_{H}(x, y)_{k} \leq T_{P}(x, y), \forall(x, y) \in I \times I$.

Proof: As above we will distinguish two cases:

Case1: In this case, it will prove that $T_{H}(x, y)_{k} \leq T_{P}(x, y)$, then

$$
\begin{aligned}
& \frac{x y}{k+(1-k)(x+y-x y)} \leq x y \\
& \Leftrightarrow \frac{1}{k+(1-k)(x+y-x y)} \leq 1 \\
& \Leftrightarrow k+(1-k)(x+y-x y) \geq 1 \\
& \Leftrightarrow-1+k+(1-k)(x+y-x y) \geq 0 \\
& \Leftrightarrow(1-k)(x+y-1-x y) \geq 0 \\
& \Leftrightarrow(1-k)(1-x)(1-y) \geq 0 .
\end{aligned}
$$

This is obvious, since $x, y \in[0,1]$ and $k \in[1,2]$.

Case2: Now we prove the relation between $T_{H}(x, y)_{k} \operatorname{and}_{B}(x, y)$.

We may see that if $x+y \leq 1$, then

$$
T_{B}(x, y)=0 \leq T_{P}(x, y) \text {. }
$$

Otherwise, if $x+y \geq 1$, and consider $T_{B}(x, y) \leq$ $T_{H}(x, y)_{k}$, then

$$
\begin{gathered}
x+y-1 \leq \frac{x y}{k+(1-k)(x+y-x y)} \\
\Leftrightarrow(x+y-1)(k+(1-k)(x+y-x y)) \leq x y \\
\Leftrightarrow(x+y-1)(k+x+y-x y-k x-k y+k x y)-x y \\
\leq 0
\end{gathered}
$$




$$
\begin{gathered}
\Leftrightarrow k x+x^{2}+x y-x^{2} y-k x^{2}-k x y+k x^{2} y+k y+x y \\
+y^{2}-x y^{2}-k x y-k y^{2}+k x y^{2}-k \\
-x-y+x y+k x+k y-k x y-x y \leq 0 \\
\Leftrightarrow-k+k x-x+x^{2}+k x-k x^{2}-y+x y+2 k y-2 k x \\
\quad+x y-x^{2}-k x y+k x^{2} y+y^{2}-x y^{2} \\
\quad-k y^{2}+k x y^{2} \leq 0 \\
\Leftrightarrow-k(1-x)-x(1-x)+k x(1-x)-y(1-x) \\
\quad+2 k y(1-x)+x y(1-x)-k x y(1-x) \\
\quad+y^{2}(1-x)-k y^{2}(1-x) \leq 0 \\
\quad-k y^{2} \leq 0 \\
\Leftrightarrow(1-x)\left(-k-x+k y+x y-k x y+y^{2}\right. \\
\Leftrightarrow(1-x)\left(-k+k y+k y-k y^{2}-x+x y+k x-k x y\right. \\
\quad-y+y^{2} \leq 0 \\
\Leftrightarrow(1-x)(-k(1-y)+k y(1-y)-x(1-y) \\
\quad+k x(1-y)-y(1-y) \leq 0 \\
\Leftrightarrow(1-x)(1-y)(-k+k y-x+k x-y) \leq 0 \\
\Leftrightarrow(1-x)(1-y)(k(x+y-1)-x-y) \leq 0 \\
\Leftrightarrow(1-x)(1-y)(x+y-1)(k-1)-1 \leq 0 . \\
\text { This is true because } \\
1-x \leq 0,1-y \leq 0 \text { and }(x+y-1)(k-1)-1 \leq 0 \\
\Leftrightarrow(x+y-1)(k-1) \leq 1 \Leftrightarrow x+y-1 \leq \frac{1}{k-1} \\
\text { Since } x+y-1 \leq 1 \leq \frac{1}{k-1}, \forall k \epsilon[1,2] . \\
\text { Hence we may conclude that } \\
T_{D}(x, y) \leq T_{B}(x, y) \leq T_{H}(x, y)_{k} \leq T_{P}(x, y) \\
\quad \leq T_{M}(x, y), \forall(x, y) \epsilon I \times I .
\end{gathered}
$$

\section{Conclusion}

Form above discussions, it conclude that T-norm Min is the strongest and T-norm Drastic product is the weakest Tnorms. Also, T-norm Algebraic product is stronger than Tnorm Hamacher product and Boundery Product. Similarly, Tnorm Dubois \& Prade product $\mathrm{T}_{\mathrm{DP}}$ is stronger than T-norm Algebraic product. Since, T-norms are using for taking suitable decision from multi-valued logic. So, the ordering of T-norms, that's means, this paper will help to take correct decision using sufficient T-norms according to their order.

\section{References}

[1] Didier Dobois, Prade Henri, FUZZY SET AND SYSTEM, THEORY AND APPLICATIONS, Academic press INC, New York.

[2] Peter Vicenik, A NOTE TO CONSTRUCTION OF T-NORMS BASED ON PSEUDO-INVERSE OF MONOTONE FUNCTIONS; Department of Mathematics, Slovak Technical University, Radlinskeho 11, 81368 Bratislava, Slovak Republic Received June 1998.

[3] George J Klir, Yuan Bo, FUZZY SETS AND FUZZY LOGIC, Theory and Applications, Prentice-Hall Inc. N.J. U.S.A. 1995.

[4] Lowen, FUZZYSET THEORY, Department of Mathematics and Computer Science, University of Antwerp; Belgium, Basic Concepts, Techniques and Bibliography, Kluwer Academic Publishers Dordeecht/Boston/London.

[5] Peter Vicenik, A NOTE ON GENERATORS OF T-NORMS; Department of Mathematics, Slovak Technical University, Radlinskeho 11, 81368 Bratislava, Slovak Republic.

[6] Matteo Bianchi, The logic of the strongest and the weakest tnorms, Fuzzy Sets Syst. 276 (2015) 31-42, http://dx.doi.org/10.1016/j.fss.2015.01. 13.

[7] Mirko Navara(2007), "Triangular norms and conforms" Scholarpedia

[8] Wladyslaw Homenda, TRIANGULAR NORMS, UNI-AND NULLNORMS,BALANCED NORMS,THE CASES OF THE HIERACHY OF ITERATIVE OPERATORS, Faculty of Mathematics and Information Science, Warsaw University of Technology, Warsaw, Poland.

[9] Mirta N.K., FUZZY SET THEORY RELATIONAL STRUCTURE USING T-NORMS AND MATHLAB, Department of Mathematics, University of Dhaka.

[10] Klement, Erich Perer; Mesiar, Radko; and Pap, Endre(2000), Triangular Norms. Dordrecht: Kluwer. ISBN 07923-6416-3. 